\title{
A Subchronic Toxicity Study of Spirulina platensis
}

\author{
Nongporn Hutadilok-Towatana, ${ }^{1,}$, Wantana ReanmongKol ${ }^{3}$, Siva Satitit ${ }^{2}$, Pharkphoom Panichayupakaranant ${ }^{4}$ \\ and Pratum RitTHISUNTHORN ${ }^{2}$ \\ ${ }^{1}$ Natural Products Research Center, Faculty of Science, Prince of Songkla University, Hat Yai, Thailand \\ ${ }^{2}$ Department of Biochemistry, Faculty of Science, Prince of Songkla University, Hat Yai, Thailand \\ ${ }^{3}$ Department of Clinical Pharmacy, Faculty of Pharmaceutical Sciences, Prince of Songkla University, Hat Yai, Thailand \\ ${ }^{4}$ Department of Pharmacognosy and Pharmaceutical Botany, Faculty of Pharmaceutical Sciences, Prince of Songkla University, Hat \\ Yai, Thailand
}

Received July 27, 2007; Accepted March 10, 2008

In this study, we examined the effects of Spirulina platensis, a multicellular filamentous blue-green algae currently used world-wide as a food supplement, upon acute and subchronic treatments to rodents. For short-term treatment, no signs of toxicity were observed within 7 days after feeding male Swiss mice at the high dose of 30 and $10 \mathrm{~g} / \mathrm{kg}$ body weight of fresh and dried $S$. platensis, respectively. For the subchronic toxicity study, two separate experiments were also performed to evaluate both forms of S. platensis. In each experiment, four groups of six Sprague-Dawley male and female rats were given fresh or dried alga at various doses by feeding daily for 12 weeks. In all instances, the consumption of algae showed no effect on behavior, food and water intake, growth or health status of these animals during the course of this investigation. The values in clinical chemistry monitored throughout the study period did not reveal significant differences between the control and treated groups. In addition, post-mortem examination found no abnormalities in the gross findings. Our results thus demonstrate for the first time that short-term and long-term consumptions of $S$. platensis, up to high feeding levels, did not produce any adverse effects in experimental animals.

Keywords: acute toxicity, subchronic toxicity, blue-green algae, Spirulina platensis

\section{Introduction}

Spirulina is an unbranched, helicoidal and filamentous blue-green algae belonging to the Division Cyanophyta. It usually grows in naturally alkaline lakes, localized in torrid zones (Jassby, 1988). Due to its high content of good-quality protein as well as being rich in vitamins, minerals and other components beneficial to health such as essential fatty acids, and antioxidant pigments like carotenoids, chlorophyll, and phycocyanin, this cyanobacterium has received much attention as a most promising and nutritious food source (Belay et al., 1993). In addition, its diverse therapeutic and pharmacological properties, such as hypocholesterolemic effect, antiviral activity, antioxidation, immune stimulation, antimutagenic potential, demonstrated both in vitro and in vivo (Charmorro et al., 1996; Khan et al., 2005), have also

*To whom correspondence should be addressed.

Email: nongporn.t@psu.ac.th promoted this alga as being a functional food, and thus at present its consumption is gaining more popularity around the world. Among the several species of spirulina available, S. maxima and S. platensis have been mostly utilized (Becker et al., 1986). They are extensively cultivated in scientifically designed algae farms and produced in large-scale systems in many countries, for human and animal consumption or for application in industry for their blue pigments. Current production of spirulina world-wide is estimated to be about 3,000 metric tons annually (Belay, 2002).

Although, toxic effects of Spirulina spp. on living organisms have been rarely found (Lightner, 1987; Fox, 1996) and this cyanobacterium has been utilized as a health food for some years without any reports of undesirable effect in humans, a more detailed evaluation of its safety for human consumption remains to be established. So far extensive toxicological tests of spirulina have been performed, mostly with S. maxima (Chamorro et al., 1985; Chamorro et al., 
1988; Chamorro et al., 1996; Salazar et al., 1996; Chamorro et al., 1997, Salazar et al., 1998). However, no study on the systemic toxicity of $S$. platensis has been reported.

Thus, both oral acute and subchronic toxicities of $S$. platensis were determined in this study in order to provide an assessment of its safety and potential for use as a dietary supplement.

\section{Materials and Methods}

Test materials Fresh S. platensis (Nordstedt) Geitler, (Oscillatoraceae) was supplied by Yord Thong 2001 Pty. Ltd. (Songkhla, Thailand). These freshly harvested algae were of a standardized quality, being part of a bulk production batch. It was identified at the Songkhla Provincial Fisheries Office where a voucher herbarium specimen (specimen no. 134.1 1916 01) has been kept at the Faculty of Pharmaceutical Sciences, Prince of Songkla University. Only one batch of S. platensis was used throughout this study. All specimens were stored in sterile containers and refrigerated prior to use within one week.

Dried algae were prepared in the oven $\left(55-60^{\circ} \mathrm{C}, 24 \mathrm{~h}\right)$ from the same batch.of fresh spirulina. They were stored at $4^{\circ} \mathrm{C}$ in sterilized sealed plastic containers and kept away from light until use.

Experimental animals Male Swiss mice with weights ranging from 30-36 g were obtained from the Animal House Facility Unit, Faculty of Science, Prince of Songkla University. They were then used for the acute toxicity tests. In the subchronic toxicity study, male and female Sprague-Dawley rats at about 5 weeks of age and weighing between 150-300 g, were purchased from the breeding colony of the National Experimental Animals Center, Nakhon Pathom, Thailand. They were acclimatized for a week after arrival. All procedures concerning animal treatments and experimentation in this study were reviewed and approved by the Institutional Committee for Ethical Use of Experimental Animals at Prince of Songkla University (approval no. 1210/713).

\section{Experimental procedures}

Acute toxicity The $50 \%$ lethal dose $\left(\mathrm{LD}_{50}\right)$ of fresh and dried S. platensis in mice was estimated by the up and down method (Bruce, 1985). Doses were adjusted up or down by a constant multiplicative factor depending on the previous outcome.

Subchronic toxicity The rats of each sex were randomly divided into 4 groups $(n=6)$ for either fresh or dried algae treatment. Each group was housed separately in an identical wire-mesh-bottomed stainless-steel cage and maintained in an air-conditioned room at $25 \pm 2{ }^{\circ} \mathrm{C}, 50-60 \%$ relative humidity and artificial illumination between 06:00 and 18:00 h. Commercial chow diets (C.P. Mice Feed, Charoen
Phokphand Group, Bangkok, Thailand) and freshly filtered water were provided ad libitum. During the 12 weeks of the experimental period, water suspensions of $S$. platensis at various doses (300, 600 and 1,200 mg/kg body weight for fresh algae treatment and 30, 60 and $120 \mathrm{mg} / \mathrm{kg}$ body weight for dried algae treatment) were orally administered (5 ml/kg body weight) to the animals in each treatment group daily. All control rats received water only $(5 \mathrm{ml} / \mathrm{kg})$ instead of a suspension of algae. The animals were observed daily for abnormalities of condition or behavior; they were weighed initially and then twice a week throughout the course of this study and before autopsy. Food and water intakes were also measured daily.

Once every 4 weeks, heparinized blood was collected by ocular bed puncture for hematology and biochemical analyses, following a 12-h fast. Packed red cell volume measurements and counts of total and differential leukocytes were performed. Plasma was also separated from the collected blood for assays of aspartate aminotransferase (AST), alanine aminotransferase (ALT), alkaline phosphatase, total and direct bilirubin, glucose, creatinine, urea nitrogen, uric acid, albumin, and total protein, according to standard clinical chemistry procedures (Burtis et al., 1999). Diagnostic kits (CPT Diagnostics, Barcelona, Spain) based on enzymatic methods were used for total cholesterol, HDL-cholesterol, LDL-cholesterol, and triglyceride assays in these plasma samples. Their $\mathrm{Na}^{+}$and $\mathrm{K}^{+}$levels were also determined by Lab Automation Model Synchron CX3 Delta (Beckman Coulter, Palo Alto, USA).

At the end of the experiments, the rats were sacrificed by cervical dislocation. An autopsy was performed during which any macroscopic abnormalities were noted. The heart, liver, spleens and kidneys were weighed immediately after removal. Samples of these organs were fixed in 10\% neutral buffered formalin and kept in that solution for further microscopic examination.

Statistical analysis All data are presented as means \pm S.E.M. Statistical evaluations were performed by the oneway ANOVA test at the 95\% confidence level using an SPSS program for Windows 11 and an LSD (Least Significance Difference) test for comparison of differences among control and treatment groups. Significance was judged at $p<0.05$.

\section{Results and Discussion}

Acute toxicity In the present study, mice were fed with fresh S. platensis at the dose of $30 \mathrm{~g} / \mathrm{kg}$ or dried alga at $10 \mathrm{~g} /$ kg did not show any signs of toxicity during 7 days of observation. There were no obvious differences between them and the control group. The gross examinations of their internal organs also revealed no pathological abnormalities. These 
results thus demonstrate that $S$. platensis is not toxic to mice after they have been exposed for short time to a high dose, and therefore indicates its potential safety for consumption.

Subchronic toxicity During the 12 weeks of this study, ingestion of fresh or dried S. platensis at any dose tested caused no significant changes on the physical appearances or clinical signs in the rats. Also, we could not detect any behavior of the spirulina-fed rats that was different from the control group. Average daily food and water intake among the groups were also similar (data not shown). The feces of rats in all treatment groups, were darkly colored and dry solids, the same as those of normal animals, indicating no abnormalities in their gastrointestinal tracts due to the consumption of spirulina. Measurements of the body weight gains over the whole study period demonstrated that the average growth rates of male rats were relatively higher than those of the females. However, the experimental rats did not show any spirulina treatment-associated effects in their body weight gains or final weights since there were no significant differences among the treated and control groups of the same sex (Figure 1). Such findings thus indicate that $S$. platensis did not alter protein, carbohydrate or fat utilization in rats and this is consistent with those previously reported in mice fed S. maxima (Salazar et al., 1998). Also the proposed probiotic effects of S. platensis (Tsuchihashi et al., 1987; de Mule et al., 1996; Parada et al., 1998) were not substantiated as there was no significant improvement in their digestion and absorption of foods.

Throughout the experimental period, there were no consistently significant differences between treated and control animals in the clinical chemistry results at any of the $S$. platensis doses tested (data not shown). Any variations observed throughout the entire period of the experiment, could have been due to biological variation among the rats. Such changes were minor, and most importantly, were not doserelated and remained within the normal ranges (Casey and King, 1980; Angkhasirisap et al., 2002). Their terminal blood values compared to their initial ones are also shown in Table 1 and Table 2. To date, a number of evidences from both animal and human studies have indicated a plasma lipid-lowering property of spirulina (Belay, 2002) and thus imply that consumption of this algae may provide potential health benefits due to the connection between hyperlipidemia and incidence of cardiovascular disease (Raza et al., 2004).
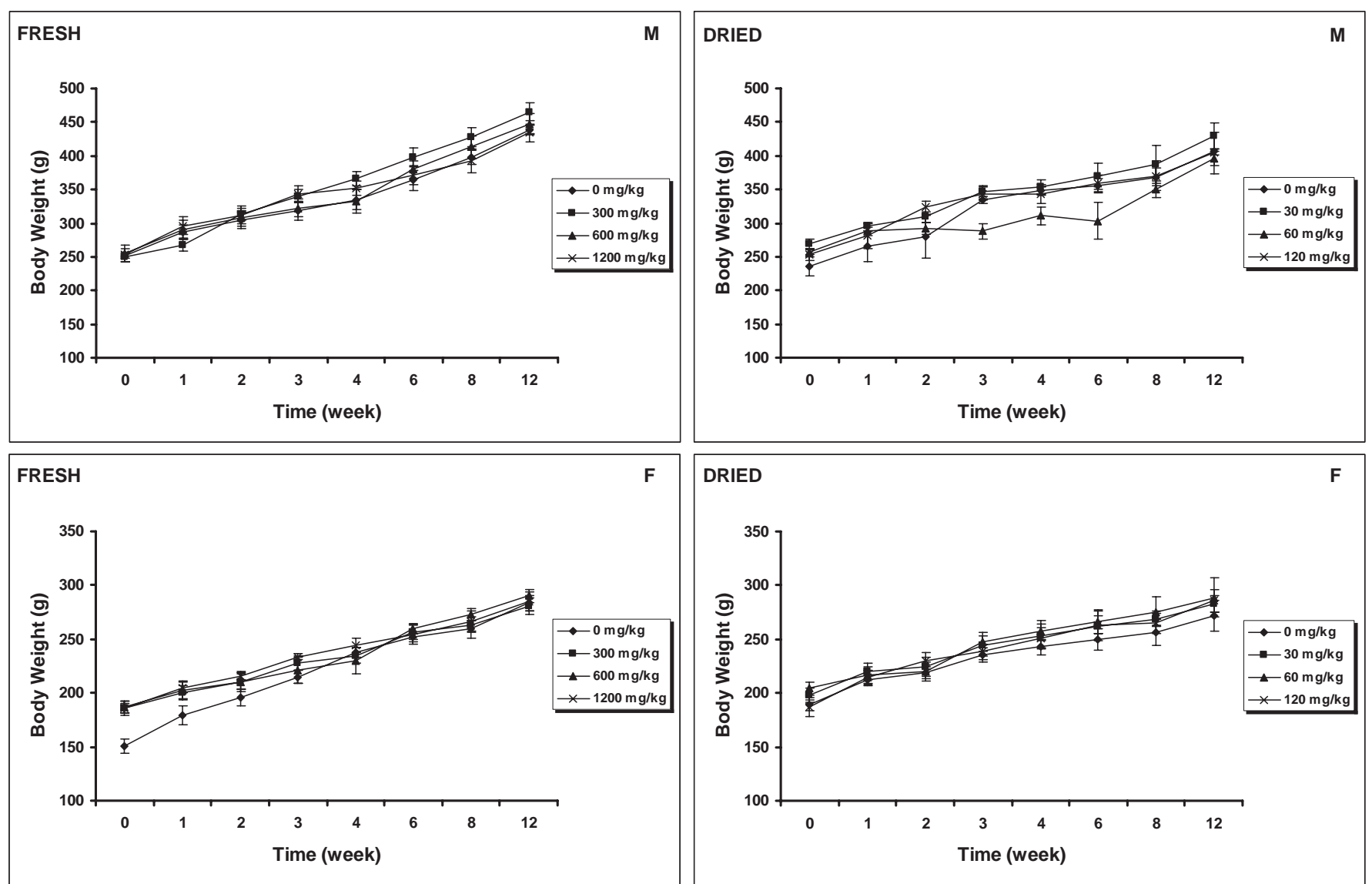

Fig. 1. Average body weights of male (M) and female (F) rats fed daily throughout 12 weeks with fresh S. platensis (FRESH, left lane) and dried S. platensis (DRIED, right lane) at various doses compared to those of the control.

Values are mean \pm S.E.M.; $n=6$. 
Table 1. Mean blood chemistry values in female and male rats treated with fresh S. platensis for 12 weeks.

Females

\begin{tabular}{|c|c|c|c|c|}
\hline \multirow[t]{2}{*}{ Parameter } & \multicolumn{4}{|c|}{ Dose of S. platensis (mg/kg) } \\
\hline & $\mathbf{0}$ & 300 & 600 & 1,200 \\
\hline \multirow[t]{2}{*}{ AST (iu/l) } & $113.72 \pm 5.56$ & $102.36 \pm 4.26$ & $92.36 \pm 5.98$ & $105.54 \pm 5.36$ \\
\hline & $(116.46 \pm 6.10)$ & $(104.64 \pm 8.38)$ & $(89.18 \pm 6.10)$ & $(118.28 \pm 5.74)$ \\
\hline \multirow[t]{2}{*}{ ALT (iu/l) } & $39.66 \pm 2.29$ & $28.92 \pm 1.22^{*}$ & $31.61 \pm 2.68^{*}$ & $34.29 \pm 2.45$ \\
\hline & $(41.56 \pm 1.34)$ & $(35.13 \pm 2.53)$ & $(36.83 \pm 1.94)$ & $(39.67 \pm 2.93)$ \\
\hline \multirow[t]{2}{*}{ ALP (iu/l) } & $33.66 \pm 3.00$ & $28.60 \pm 1.38$ & $29.62 \pm 3.42$ & $33.66 \pm 3.48$ \\
\hline & $(42.70 \pm 1.14)$ & $(38.56 \pm 1.66)$ & $(38.24 \pm 1.48)$ & $(35.68 \pm 2.94)$ \\
\hline \multirow[t]{2}{*}{ Bilirubin (mg/dl) } & $0.11 \pm 0.01$ & $0.10 \pm 0.01$ & $0.10 \pm 0.01$ & $0.12 \pm 0.01$ \\
\hline & $(0.10 \pm 0.03)$ & $(0.10 \pm 0.01)$ & $(0.12 \pm 0.01)$ & $(0.10 \pm 0.01)$ \\
\hline \multirow[t]{2}{*}{ Protein (g/dl) } & $6.41 \pm 0.05$ & $6.64 \pm 0.16$ & $6.53 \pm 0.18$ & $6.60 \pm 0.20$ \\
\hline & $(5.24 \pm 0.13)$ & $(5.42 \pm 0.13)$ & $(5.17 \pm 0.16)$ & $(5.63 \pm 0.09 \dagger)$ \\
\hline \multirow[t]{2}{*}{ Albumin (g/dl) } & $4.09 \pm 0.10$ & $4.33 \pm 0.07$ & $4.39 \pm 0.14^{*}$ & $4.36 \pm 0.08$ \\
\hline & $(3.67 \pm 0.26)$ & $(4.02 \pm 0.05)$ & $(4.02 \pm 0.07)$ & $(4.14 \pm 0.09 \dagger)$ \\
\hline \multirow[t]{2}{*}{ Cholesterol (mg/dl) } & $80.97 \pm 3.47$ & $80.56 \pm 1.93$ & $90.00 \pm 5.02$ & $86.11 \pm 3.47$ \\
\hline & $(87.88 \pm 6.18)$ & $(104.55 \pm 3.09 \dagger)$ & $(106.06 \pm 3.09 \dagger)$ & $(106.06 \pm 3.09 \dagger)$ \\
\hline \multirow[t]{2}{*}{ TG (mg/dl) } & $40.00 \pm 3.54$ & $40.00 \pm 4.42$ & $45.33 \pm 7.08$ & $40.00 \pm 6.19$ \\
\hline & $(50.00 \pm 5.31)$ & $(50.00 \pm 4.42)$ & $(40.00 \pm 2.65)$ & $(45.83 \pm 3.54)$ \\
\hline \multirow[t]{2}{*}{ HDL-C (mg/dl) } & $51.39 \pm 2.70$ & $52.78 \pm 1.54$ & $56.67 \pm 4.25$ & $58.33 \pm 3.86$ \\
\hline & $(44.64 \pm 1.93)$ & $(51.19 \pm 2.32)$ & $(53.57 \pm 3.09+)$ & $(57.14 \pm 2.70 \dagger)$ \\
\hline \multirow[t]{2}{*}{ LDL-C (mg/dl) } & $21.58 \pm 1.78$ & $19.78 \pm 3.03$ & $24.27 \pm 2.09$ & $19.78 \pm 1.83$ \\
\hline & $(32.24 \pm 4.80)$ & $(43.35 \pm 1.88 \dagger)$ & $(44.49 \pm 2.47 \dagger)$ & $(39.75 \pm 3.12 \dagger)$ \\
\hline \multirow[t]{2}{*}{ Glucose (mg/dl) } & $100.00 \pm 5.77$ & $116.67 \pm 4.94^{*}$ & $104.00 \pm 2.45$ & $103.33 \pm 5.57$ \\
\hline & $(138.89 \pm 2.48)$ & $(127.78 \pm 5.56)$ & $(131.48 \pm 3.41)$ & $(111.11 \pm 5.74 \dagger)$ \\
\hline \multirow[t]{2}{*}{ BUN (mg/dl) } & $28.06 \pm 0.74$ & $25.88 \pm 1.26$ & $29.26 \pm 1.46$ & $32.84 \pm 1.34^{*}$ \\
\hline & $(30.50 \pm 1.98)$ & $(25.98 \pm 1.14 \dagger)$ & $(28.24 \pm 1.42)$ & $(33.06 \pm 0.86)$ \\
\hline \multirow[t]{2}{*}{ Creatinine (mg/dl) } & $0.43 \pm 0.05$ & $0.46 \pm 0.04$ & $0.47 \pm 0.05$ & $0.46 \pm 0.04$ \\
\hline & $(0.45 \pm 0.06)$ & $(0.45 \pm 0.05)$ & $(0.42 \pm 0.06)$ & $(0.44 \pm 0.08)$ \\
\hline \multirow[t]{2}{*}{ Uric Acid (mg/dl) } & $0.81 \pm 0.07$ & $0.77 \pm 0.13$ & $0.74 \pm 0.07$ & $0.74 \pm 0.08$ \\
\hline & $(0.81 \pm 0.07)$ & $(0.71 \pm 0.07)$ & $(0.75 \pm 0.07)$ & $(0.86 \pm 0.07)$ \\
\hline
\end{tabular}

\begin{tabular}{|c|c|c|c|c|}
\hline \multicolumn{5}{|c|}{ Males } \\
\hline \multirow[t]{2}{*}{ Parameter } & \multicolumn{4}{|c|}{ Dose of S. platensis (mg/kg) } \\
\hline & $\mathbf{0}$ & 300 & 600 & 1,200 \\
\hline \multirow[t]{2}{*}{ AST (iu/l) } & $93.28 \pm 3.94$ & $89.86 \pm 5.28$ & $84.18 \pm 6.34$ & $88.72 \pm 4.26$ \\
\hline & $(100.10 \pm 5.48)$ & $(100.10 \pm 7.04)$ & $(86.46 \pm 6.42)$ & $(100.10 \pm 9.96)$ \\
\hline \multirow[t]{2}{*}{ ALT (iu/l) } & $28.43 \pm 2.74$ & $25.15 \pm 1.55$ & $31.71 \pm 1.27$ & $25.15 \pm 1.16$ \\
\hline & $(32.87 \pm 1.03)$ & $(36.83 \pm 1.94)$ & $(34.00 \pm 1.46)$ & $(34.00 \pm 2.07)$ \\
\hline \multirow[t]{2}{*}{ ALP (iu/l) } & $48.24 \pm 2.06$ & $43.08 \pm 1.64$ & $43.08 \pm 1.64$ & $52.72 \pm 4.40$ \\
\hline & $(51.22+2.88)$ & $(60.88+2.76+)$ & $(51.56 \pm 1.88)$ & $(59.84+2.12)$ \\
\hline \multirow[t]{2}{*}{ Bilirubin (mg/dl) } & $0.10 \pm 0.01$ & $0.11 \pm 0.01$ & $0.12 \pm 0.01$ & $0.09 \pm 0.01$ \\
\hline & $(0.09 \pm 0.01)$ & $(0.08 \pm 0.01)$ & $(0.09 \pm 0.01)$ & $(0.11 \pm 0.01)$ \\
\hline \multirow[t]{2}{*}{ Protein (g/dl) } & $6.56 \pm 0.07$ & $6.26 \pm 0.21$ & $6.44 \pm 0.08$ & $6.64 \pm 0.13$ \\
\hline & $(5.03 \pm 0.17)$ & $(5.03 \pm 0.04)$ & $(5.07 \pm 0.12)$ & $(5.35 \pm 0.13)$ \\
\hline \multirow[t]{2}{*}{ Albumin (g/dl) } & $4.04 \pm 0.05$ & $4.04 \pm 0.07$ & $4.01 \pm 0.06$ & $3.92 \pm 0.04$ \\
\hline & $(3.91 \pm 0.10)$ & $(3.79 \pm 0.09)$ & $(3.76 \pm 0.10)$ & $(3.94 \pm 0.10)$ \\
\hline \multirow[t]{2}{*}{ Cholesterol (mg/dl) } & $72.22 \pm 2.70$ & $71.67 \pm 1.93$ & $70.00 \pm 1.93$ & $61.11 \pm 2.70^{*}$ \\
\hline & $(81.82 \pm 2.70)$ & $(74.24 \pm 1.54)$ & $(81.82 \pm 2.32)$ & $(83.33 \pm 3.47)$ \\
\hline \multirow[t]{2}{*}{ TG (mg/dl) } & $51.11 \pm 2.65$ & $62.22 \pm 4.42$ & $43.33 \pm 6.19$ & $48.89 \pm 2.65$ \\
\hline & $(50.00 \pm 3.54)$ & $(42.50 \pm 5.31)$ & $(41.67 \pm 4.42)$ & $(45.83 \pm 6.19)$ \\
\hline \multirow[t]{2}{*}{ HDL-C (mg/dl) } & $43.06 \pm 2.70$ & $41.67 \pm 2.32$ & $45.00 \pm 1.93$ & $38.89 \pm 1.93$ \\
\hline & $(45.71 \pm 1.93)$ & $(40.48 \pm 1.54)$ & $(44.05 \pm 2.32)$ & $(45.24 \pm 1.54)$ \\
\hline \multirow[t]{2}{*}{ LDL-C (mg/dl) } & $18.94 \pm 2.80$ & $17.56 \pm 1.83$ & $16.33 \pm 1.40$ & $12.44 \pm 1.89$ \\
\hline & $(26.10 \pm 2.35)$ & $(25.27 \pm 1.67)$ & $(29.44 \pm 1.22)$ & $(28.93 \pm 3.86)$ \\
\hline \multirow[t]{2}{*}{ Glucose (mg/dl) } & $13.00 \pm 6.83$ & $128.00 \pm 3.74$ & $130.00 \pm 8.94$ & $116.67 \pm 8.03$ \\
\hline & $(116.67 \pm 5.56)$ & $(122.22 \pm 4.06)$ & $(138.89 \pm 4.76 \dagger)$ & $(125.93 \pm 5.49)$ \\
\hline \multirow[t]{2}{*}{ BUN (mg/dl) } & $30.34 \pm 0.50$ & $31.34 \pm 3.56$ & $28.06 \pm 0.74$ & $28.36 \pm 0.68$ \\
\hline & $(25.42+0.76)$ & $(24.40+1.66)$ & $(22.38+0.82)$ & $(27.12+1.50)$ \\
\hline \multirow[t]{2}{*}{ Creatinine (mg/dl) } & $0.59 \pm 0.05$ & $0.57 \pm 0.05$ & $0.56 \pm 0.07$ & $0.62 \pm 0.06$ \\
\hline & $(0.62 \pm 0.07)$ & $(0.58 \pm 0.04)$ & $(0.54 \pm 0.04)$ & $(0.49 \pm 0.06)$ \\
\hline \multirow[t]{2}{*}{ Uric Acid (mg/dl) } & $0.86 \pm 0.07$ & $0.80 \pm 0.07$ & $0.67 \pm 0.07$ & $0.77 \pm 0.07$ \\
\hline & $(0.86 \pm 0.07)$ & $(0.73 \pm 0.07)$ & $(0.80 \pm 0.07)$ & $(0.73 \pm 0.07)$ \\
\hline
\end{tabular}

Values are Mean + S.E.M.; $\mathrm{n}=6$

Each corresponding initial value (week 0 ) is shown in brackets.

$* p<0.05$ vs final control (week 12). $\dagger p<0.05 v s$ initial control (week 0 ).

AST $=$ Aspartate Transaminase, ALT $=$ Alanine Transaminase, ALP $=$ Alkaline Phosphatase, TG $=$ Triglyceride, HDL-C = HDL-cholesterol, LDL-C = LDL-cholesterol, BUN = urea nitrogen.

It has been postulated that any decrease in cholesterol levels might be attributed to the increased fecal excretion of cholesterol or to the presence of eicosapentaenoic acid in the algae (Lacaz-Ruiz and Mos, 1990), while the hypotriglyceridemic
Table 2. Mean blood chemistry values in female and male rats treated with dried S. platensis for 12 weeks.

Females

\begin{tabular}{|c|c|c|c|c|}
\hline \multirow[t]{2}{*}{ Parameter } & \multicolumn{4}{|c|}{ Dose of S. platensis (mg/kg) } \\
\hline & $\mathbf{0}$ & 30 & 60 & 120 \\
\hline \multirow[t]{2}{*}{ AST (iu/l) } & $100.88 \pm 8.78$ & $91.98 \pm 7.60$ & $106.70 \pm 6.02$ & $91.12 \pm 5.56$ \\
\hline & $(107.26 \pm 6.72)$ & $(94.44 \pm 7.88)$ & $(103.26 \pm 4.38)$ & $(98.02 \pm 7.04)$ \\
\hline \multirow[t]{2}{*}{ ALT (iu/l) } & $33.05 \pm 2.04$ & $38.05 \pm 1.82$ & $36.05 \pm 2.33$ & $41.38 \pm 1.29 *$ \\
\hline & $(35.90 \pm 1.09)$ & $(44.07 \pm 4.00)$ & $(39.17 \pm 1.89)$ & $(46.79 \pm 1.54)$ \\
\hline \multirow[t]{2}{*}{ ALP (iu/l) } & $29.96 \pm 2.50$ & $27.08 \pm 1.94$ & $24.32 \pm 1.10$ & $23.96 \pm 3.12$ \\
\hline & $(33.50 \pm 1.48)$ & $(39.82 \pm 2.44)$ & $(37.22 \pm 2.08)$ & $(31.16 \pm 2.12)$ \\
\hline \multirow[t]{2}{*}{ Bilirubin (mg/dl) } & $0.11 \pm 0.004$ & $0.11 \pm 0.01$ & $0.10 \pm 0.01$ & $0.10 \pm 0.003$ \\
\hline & $(0.12 \pm 0.01)$ & $(0.10 \pm 0.01)$ & $(0.10 \pm 0.01)$ & $(0.11 \pm 0.01)$ \\
\hline \multirow[t]{2}{*}{ Protein (g/dl) } & $5.43+0.08$ & $5.45+0.12$ & $5.13+0.13$ & $5.45+0.06$ \\
\hline & $(5.56 \pm 0.10)$ & $(4.55 \pm 0.21 \dagger)$ & $(4.38 \pm 0.12 \dagger)$ & $(4.38 \pm 0.15 \dagger)$ \\
\hline \multirow[t]{2}{*}{ Albumin (g/dl) } & $3.75 \pm 0.16$ & $3.74 \pm 0.17$ & $3.83 \pm 0.14$ & $4.43 \pm 0.11^{*}$ \\
\hline & $(3.35 \pm 0.13)$ & $(2.65 \pm 0.11 \dagger)$ & $(2.68 \pm 0.09 \dagger)$ & $(2.51 \pm 0.10 \dagger)$ \\
\hline \multirow[t]{2}{*}{ Cholesterol (mg/dl) } & $77.08 \pm 3.99$ & $81.67 \pm 4.85$ & $93.06 \pm 5.86^{*}$ & $85.00 \pm 4.08$ \\
\hline & $(93.48 \pm 1.77)$ & $(89.28 \pm 4.73)$ & $(91.30 \pm 2.51)$ & $(95.65 \pm 5.80)$ \\
\hline \multirow[t]{2}{*}{ TG (mg/dl) } & $32.35 \pm 5.63$ & $41.18 \pm 3.03$ & $44.12 \pm 2.40$ & $35.29 \pm 4.29$ \\
\hline & $(38.45 \pm 8.38)$ & $(29.70 \pm 2.95)$ & $(32.83 \pm 2.55)$ & $(47.94 \pm 2.95)$ \\
\hline \multirow[t]{2}{*}{ HDL-C (mg/dl) } & $18.52 \pm 1.60$ & $14.44 \pm 1.36^{*}$ & $17.78 \pm 1.01$ & $14.44 \pm 1.36^{*}$ \\
\hline & $(18.75 \pm 1.24)$ & $(19.48 \pm 1.12)$ & $(18.75 \pm 2.28)$ & $(18.75 \pm 1.96)$ \\
\hline \multirow[t]{2}{*}{ LDL-C (mg/dl) } & $52.09 \pm 2.67$ & $58.99 \pm 5.93$ & $66.45 \pm 8.12$ & $63.50 \pm 4.04$ \\
\hline & $(67.04 \pm 2.12)$ & $(63.86 \pm 5.02)$ & $(65.99 \pm 1.09)$ & $(67.32 \pm 1.31)$ \\
\hline \multirow[t]{2}{*}{ Glucose (mg/dl) } & $130.00 \pm 5.77$ & $116.00 \pm 3.99$ & $103.33 \pm 6.15^{*}$ & $140.00 \pm 6.31$ \\
\hline & $(127.27 \pm 7.42)$ & $(123.64 \pm 6.21)$ & $(90.91 \pm 5.25 \dagger)$ & $(124.24 \pm 5.59)$ \\
\hline \multirow[t]{2}{*}{ BUN (mg/dl) } & $23.94 \pm 1.40$ & $27.04 \pm 2.10$ & $19.16 \pm 1.26$ & $25.92 \pm 1.38$ \\
\hline & $(26.42 \pm 2.18)$ & $(24.52 \pm 1.54)$ & $(26.42 \pm 1.68)$ & $(27.16 \pm 1.68)$ \\
\hline \multirow[t]{2}{*}{ Creatinine (mg/dl) } & $0.45 \pm 0.05$ & $0.44 \pm 0.03$ & $0.44 \pm 0.06$ & $0.41 \pm 0.06$ \\
\hline & $(0.41 \pm 0.05)$ & $(0.45 \pm 0.07)$ & $(0.45 \pm 0.07)$ & $(0.41 \pm 0.05)$ \\
\hline \multirow[t]{2}{*}{ Uric Acid (mg/dl) } & $0.74 \pm 0.06$ & $0.73 \pm 0.07$ & $0.78 \pm 0.05$ & $0.81 \pm 0.05$ \\
\hline & $(0.77 \pm 0.06)$ & $(0.75 \pm 0.05)$ & $(0.75 \pm 0.05)$ & $(0.78 \pm 0.05)$ \\
\hline
\end{tabular}

\begin{tabular}{|c|c|c|c|c|}
\hline \multicolumn{5}{|c|}{ Males } \\
\hline \multirow[t]{2}{*}{ Parameter } & \multicolumn{4}{|c|}{ Dose of S. platensis (mg/kg) } \\
\hline & 0 & 30 & 60 & 120 \\
\hline \multirow{2}{*}{ AST (iu/l) } & $80.32 \pm 4.90$ & $71.42 \pm 7.94$ & $75.88 \pm 8.00$ & $74.22 \pm 8.50$ \\
\hline & $(83.52 \pm 10.38)$ & $(78.50 \pm 6.24)$ & $(67.54 \pm 8.16)$ & $(71.18 \pm 8.32)$ \\
\hline \multirow[t]{2}{*}{ ALT (iu/l) } & $24.72 \pm 1.29$ & $27.22 \pm 1.54$ & $32.05 \pm 2.91$ & $33.05 \pm 2.04 *$ \\
\hline & $(26.09 \pm 1.89)$ & $(19.55 \pm 1.89 \dagger)$ & $(29.36 \pm 2.18)$ & $(28.05 \pm 2.23)$ \\
\hline \multirow[t]{2}{*}{ ALP (iu/l) } & $36.46 \pm 2.84$ & $36.46 \pm 3.00$ & $39.60 \pm 2.08$ & $37.34 \pm 3.12$ \\
\hline & $(43.80 \pm 1.48)$ & $(41.22 \pm 2.10)$ & $(38.64 \pm 1.48)$ & $(39.94 \pm 2.02)$ \\
\hline \multirow[t]{2}{*}{ Bilirubin (mg/dl) } & $0.09 \pm 0.00$ & $0.10 \pm 0.01$ & $0.08 \pm 0.01$ & $0.09 \pm 0.01$ \\
\hline & $(0.09 \pm 0.01)$ & $(0.09 \pm 0.01)$ & $(0.10 \pm 0.01)$ & $(0.09 \pm 0.01)$ \\
\hline \multirow[t]{2}{*}{ Protein (g/dl) } & $5.38 \pm 0.08$ & $5.35 \pm 0.11$ & $4.99 \pm 0.08^{*}$ & $5.24 \pm 0.05$ \\
\hline & $(5.40 \pm 0.19)$ & $(4.38 \pm 0.15 \dagger)$ & $(4.27 \pm 0.07 \dagger)$ & $(4.38 \pm 0.09 \dagger)$ \\
\hline \multirow[t]{2}{*}{ Albumin (g/dl) } & $2.91 \pm 0.04$ & $2.90 \pm 0.08$ & $3.00 \pm 0.07$ & $3.34 \pm 0.12 *$ \\
\hline & $(2.79 \pm 0.07)$ & $(2.36 \pm 0.07 \dagger)$ & $(2.34 \pm 0.05 \dagger)$ & $(2.36 \pm 0.09 \dagger)$ \\
\hline \multirow[t]{2}{*}{ Cholesterol (mg/dl) } & $68.33 \pm 1.66$ & $76.39 \pm 3.98$ & $71.67 \pm 3.33$ & $73.61 \pm 4.52$ \\
\hline & $(75.13 \pm 3.64)$ & $(66.67 \pm 1.83)$ & $(73.91 \pm 8.32)$ & $(76.81+5.69)$ \\
\hline \multirow[t]{2}{*}{ TG (mg/dl) } & $49.41 \pm 4.39$ & $56.47 \pm 3.53$ & $47.06 \pm 3.71$ & $54.90 \pm 4.96$ \\
\hline & $(55.00 \pm 2.79)$ & $(50.63 \pm 2.65)$ & $(50.00 \pm 5.10)$ & $(50.42+3.24)$ \\
\hline \multirow[t]{2}{*}{ HDL-C (mg/dl) } & $28.89 \pm 2.08$ & $28.70 \pm 0.93$ & $28.89 \pm 2.08$ & $27.78 \pm 1.43$ \\
\hline & $(31.25 \pm 1.80)$ & $(28.13 \pm 1.62)$ & $(33.75 \pm 1.40)$ & $(31.25 \pm 1.61)$ \\
\hline \multirow[t]{2}{*}{ LDL-C (mg/dl) } & $29.56 \pm 3.85$ & $36.39 \pm 3.75$ & $33.37 \pm 2.10$ & $34.85 \pm 3.97$ \\
\hline & $(32.88 \pm 4.63)$ & $(28.42 \pm 3.37)$ & $(30.16 \pm 2.98)$ & $(35.48 \pm 4.97)$ \\
\hline \multirow[t]{2}{*}{ Glucose (mg/dl) } & $124.00 \pm 7.47$ & $120.00 \pm 5.16$ & $124.00 \pm 3.99$ & $112.00 \pm 4.47$ \\
\hline & $(118.18 \pm 5.25)$ & $(127.27 \pm 7.42)$ & $(101.82 \pm 4.06)$ & $(112.12 \pm 5.59)$ \\
\hline \multirow[t]{2}{*}{ BUN (mg/dl) } & $21.40 \pm 1.12$ & $23.48 \pm 1.74$ & $19.16 \pm 1.38$ & $22.54 \pm 1.46$ \\
\hline & $(22.64 \pm 1.26)$ & $(18.12 \pm 1.68)$ & $(25.66 \pm 1.68)$ & $(22.64 \pm 2.18)$ \\
\hline \multirow[t]{2}{*}{ Creatinine (mg/dl) } & $0.60 \pm 0.09$ & $0.63 \pm 0.10$ & $0.87 \pm 0.08$ & $0.60 \pm 0.03$ \\
\hline & $(0.62 \pm 0.06)$ & $(0.63 \pm 0.05)$ & $(0.58 \pm 0.08)$ & $(0.55 \pm 0.08)$ \\
\hline \multirow[t]{2}{*}{ Uric Acid (mg/dl) } & $0.75 \pm 0.09$ & $0.79 \pm 0.05$ & $0.83 \pm 0.05$ & $0.75 \pm 0.05$ \\
\hline & $(0.79 \pm 0.05)$ & $(0.75 \pm 0.10)$ & $(0.78 \pm 0.05)$ & $(0.73+0.06)$ \\
\hline
\end{tabular}

Values are Mean + S.E.M.; $\mathrm{n}=6$.

Each corresponding initial value (week 0 ) is shown in brackets.

$* p<0.05$ vs final control (week 12). $\dagger p<0.05$ vs initial control (week 0 ).

AST $=$ Aspartate Transaminase, ALT $=$ Alanine Transaminase, ALP $=$ Alkaline Phosphatase, $\mathrm{TG}=$ Triglyceride, HDL-C = HDL-cholesterol, LDL-C = LDL-cholesterol, BUN = urea nitrogen.

effect might be through its effect on the metabolism of lipoproteins (Itawa et al., 1990). Surprisingly, in this study, we did not observe any significant alterations of blood lipid profiles in any of our treated animals relative to the controls 
(Table 1 and Table 2). At the beginning of study (week 0), however, all of the three female fresh-spirulina treatment groups had their average levels of total cholesterol and LDL-C significantly higher than the normal control (Table 1). Such elevations in both lipid parameters were due to some unknown cause and were not found in the other periods (week 4, week 8, and week 12) (data not shown). Consequently, the differences between the initial and the final total cholesterol and LDL-C data in each female treated group as shown in Table 1 were interpreted as the variation effects rather than the results of spirulina treatment. It is noteworthy, also, that pronounced cholesterol reduction and related effects of spirulina have been reported mostly in subjects with elevated blood lipid levels (Belay, 2002). Thus, hyperlipidemia may be a more sensitive experimental model than the one having a normal blood lipid range, in this regard. Since spirulina has a high $\mathrm{K}^{+}$content (Belay et al., 1993), long-term consumption of this alga especially in a large amount would probably lead to hyperkeliemia and its related disorders. In our subchronic toxicity study, we could not detect any in- creases in blood $\mathrm{K}^{+}$levels in any treated animals throughout the 12 weeks of investigation. All measured values were within the same range of 149-152 mmole/l (data not shown). This indicates no accumulation of $\mathrm{K}^{+}$in the rat's circulation due to continuous intake of $S$. platensis even at high dosage.

To determine if rats treated with fresh S. platensis had any intravascular effects and bone marrow activity, hematological examinations were performed. As presented in Table 3 , the average hematocrit values in all female groups appear to be lower than those in the males. Although, feeding the rats with fresh algae, at any of the three doses for 12 weeks, did cause significant increases in this hematological parameter, the effects were slight and not dose-related. During the course of study, both total and differential WBC counts were also similar to normal values (Table 3), with lymphocytes being the major WBC populations detected (Inala et al., 2002). However, at the end of the experimental period, lymphocyte ratios in all spirulina-receiving groups became lower than those of the control (Table 3). This was mainly due to an increased number of neutrophils and monocytes. An increase

Table 3. Mean hematological values in female and male rats treated with fresh S. platensis for 12 weeks.

\section{Females}

\begin{tabular}{llllll}
\hline \multirow{2}{*}{ Dose $(\mathbf{m g} / \mathbf{k g})$} & Hct (\%) & WBC $(\mathbf{x ~ 1 0} / \mu \mathbf{l})$ & \multicolumn{3}{c}{ Differential Leucocytes (\%) } \\
\cline { 3 - 6 } & & & $\mathbf{( N )}$ & $\mathbf{( L )}$ & $\mathbf{( M )}$ \\
\hline $\mathbf{0}$ & $34.60 \pm 1.18^{*}$ & $3.15 \pm 0.27$ & $0.33 \pm 0.21^{*}$ & $95.67 \pm 0.99$ & $4.00 \pm 1.09^{*}$ \\
& $(29.33 \pm 0.85)$ & $(3.13 \pm 0.09)$ & $(1.67 \pm 0.47)$ & $(98.33 \pm 0.47)$ & $(0)$ \\
\hline $\mathbf{3 0 0}$ & $35.17 \pm 1.35^{*}$ & $2.91 \pm 0.18$ & $2.00 \pm 0.86$ & $93.67 \pm 3.15^{*}$ & $4.33 \pm 2.33^{*}$ \\
& $(31.17 \pm 0.75)$ & $(3.00 \pm 0.12)$ & $(2.67 \pm 0.56)$ & $(97.33 \pm 0.56)$ & $(0)$ \\
\hline $\mathbf{6 0 0}$ & $37.40 \pm 1.10^{*}$ & $2.91 \pm 0.24$ & $10.80 \pm 6.04^{*}$ & $85.20 \pm 5.65^{*}$ & $4.00 \pm 0.82^{*}$ \\
& $(30.33 \pm 0.92)$ & $(2.82 \pm 0.11)$ & $(4.00 \pm 1.03)$ & $(96.00 \pm 1.03)$ & $(0)$ \\
\hline $\mathbf{1 , 2 0 0}$ & $37.00 \pm 0.58^{*}$ & $2.54 \pm 0.28$ & $11.50 \pm 1.84^{*}$ & $87.00 \pm 2.18^{*}$ & $1.50 \pm 0.43^{*}$ \\
& $(30.83 \pm 0.75)$ & $(2.41 \pm 0.11)$ & $(4.33 \pm 1.05)$ & $(95.33 \pm 0.96)$ & $(0.33 \pm 0.33)$ \\
\hline
\end{tabular}

Males

\begin{tabular}{llllll}
\hline \multirow{2}{*}{ Dose $(\mathbf{m g} / \mathbf{k g})$} & Hct (\%) & WBC $(\times \mathbf{~ 1 0} / \mu \mathbf{l})$ & \multicolumn{3}{c}{ Differential Leucocytes (\%) } \\
\cline { 4 - 6 } & & & $\mathbf{( N )}$ & $\mathbf{( L )}$ & $\mathbf{( M )}$ \\
\hline $\mathbf{0}$ & $40.83 \pm 0.83^{*}$ & $3.84 \pm 0.15$ & $1.33 \pm 0.42^{*}$ & $95.17 \pm 1.30$ & $3.50 \pm 0.96^{*}$ \\
& $(32.50 \pm 0.72)$ & $(3.89 \pm 1.12)$ & $(2.50 \pm 0.62)$ & $(97.50 \pm 0.62)$ & $(0)$ \\
\hline $\mathbf{3 0 0}$ & $39.20 \pm 0.45^{*}$ & $3.76 \pm 0.15^{*}$ & $2.20 \pm 0.89$ & $85.20 \pm 1.67 *$ & $12.60 \pm 1.24^{*}$ \\
& $(30.50 \pm 0.85)$ & $(4.00 \pm 0.09)$ & $(1.67 \pm 1.11)$ & $(98.33 \pm 1.11)$ & $(0)$ \\
\hline $\mathbf{6 0 0}$ & $39.60 \pm 0.68^{*}$ & $3.46 \pm 0.28$ & $6.60 \pm 1.36^{*}$ & $90.20 \pm 1.02^{*}$ & $3.20 \pm 0.53^{*}$ \\
& $(32.83 \pm 0.79)$ & $(3.51 \pm 1.34)$ & $(1.67 \pm 0.62)$ & $(98.33 \pm 0.62)$ & $(0)$ \\
\hline $\mathbf{1 , 2 0 0}$ & $37.67 \pm 0.95^{*}$ & $3.73 \pm 0.23$ & $15.33 \pm 2.67^{*}$ & $79.50 \pm 3.29^{*}$ & $5.17 \pm 0.95^{*}$ \\
& $(34.67 \pm 0.67)$ & $(3.92 \pm 0.45)$ & $(3.50 \pm 1.43)$ & $(96.33 \pm 1.52)$ & $(0.17 \pm 0.17)$ \\
\hline
\end{tabular}

Values are Mean \pm S.E.M.; $\mathrm{n}=6$. $* p<0.05$ vs its initial value.

Each corresponding initial value (week 0 ) is shown in brackets.

Hct = Hematocrit, $\mathrm{N}=$ Neutrophil, $\mathrm{L}$ = Lymphocyte, $\mathrm{M}$ = Monocyte 
Table 4. Mean organ weights in female and male rats treated with S. platensis for 12 weeks.

Females

\begin{tabular}{lllll}
\hline \multirow{2}{*}{$\begin{array}{l}\text { Dresh Spirulina } \\
\text { Dose (mg/kg) }\end{array}$} & Liver & Kidney & Spleen & Heart \\
\cline { 2 - 5 } $\mathbf{0}$ & $6.50 \pm 0.09$ & $1.67 \pm 0.04$ & $0.66 \pm 0.01$ & $0.94 \pm 0.04$ \\
$\mathbf{3 0 0}$ & $6.13 \pm 0.15$ & $1.52 \pm 0.06$ & $0.66 \pm 0.04$ & $0.99 \pm 0.03$ \\
$\mathbf{6 0 0}$ & $6.52 \pm 0.28$ & $1.57 \pm 0.03$ & $0.71 \pm 0.01$ & $0.93 \pm 0.01$ \\
$\mathbf{1 , 2 0 0}$ & $6.53 \pm 0.26$ & $1.58 \pm 0.04$ & $0.70 \pm 0.04$ & $0.99 \pm 0.03$ \\
\hline Dried Spirulina & & \multicolumn{3}{c}{ Organ Weight (g) } \\
\cline { 2 - 5 } Dose (mg/kg) & Liver & Kidney & Spleen & Heart \\
\hline $\mathbf{0}$ & $6.34 \pm 0.73$ & $1.62 \pm 0.17$ & $0.56 \pm 0.10$ & $0.94 \pm 0.08$ \\
\hline $\mathbf{3 0}$ & $6.27 \pm 0.45$ & $1.64 \pm 0.10$ & $0.67 \pm 0.08$ & $0.99 \pm 0.08$ \\
\hline $\mathbf{6 0}$ & $7.56 \pm 1.47$ & $1.79 \pm 0.21$ & $0.69 \pm 0.12$ & $1.06 \pm 0.15$ \\
\hline $\mathbf{1 2 0}$ & $6.91 \pm 0.65$ & $1.67 \pm 0.20$ & $0.60 \pm 0.02$ & $1.00 \pm 0.12$ \\
\hline
\end{tabular}

Males

\begin{tabular}{|c|c|c|c|c|}
\hline \multirow{2}{*}{$\begin{array}{l}\text { Fresh Spirulina } \\
\text { Dose (mg/kg) }\end{array}$} & \multicolumn{4}{|c|}{ Organ Weight (g) } \\
\hline & Liver & Kidney & Spleen & Heart \\
\hline $\mathbf{0}$ & $10.23 \pm 0.33$ & $2.37 \pm 0.09$ & $0.79 \pm 0.04$ & $1.42 \pm 0.03$ \\
\hline 300 & $10.77 \pm 0.56$ & $2.68 \pm 0.13$ & $0.87 \pm 0.03$ & $1.67 \pm 0.10$ \\
\hline 600 & $10.74 \pm 0.49$ & $2.61 \pm 0.12$ & $0.82 \pm 0.04$ & $1.49 \pm 0.07$ \\
\hline 1,200 & $10.11 \pm 0.79$ & $2.47 \pm 0.08$ & $0.81 \pm 0.02$ & $1.48 \pm 0.08$ \\
\hline Dried Spirulina & \multicolumn{4}{|c|}{ Organ Weight (g) } \\
\hline Dose (mg/kg) & Liver & Kidney & Spleen & Heart \\
\hline $\mathbf{0}$ & $10.07 \pm 0.37$ & $2.53 \pm 0.07$ & $0.77 \pm 0.03$ & $1.37 \pm 0.05$ \\
\hline 30 & $10.35 \pm 0.46$ & $2.47 \pm 0.10$ & $0.76 \pm 0.05$ & $1.68 \pm 0.13$ \\
\hline 60 & $9.03 \pm 0.37$ & $2.34 \pm 0.04$ & $0.80 \pm 0.04$ & $1.28 \pm 0.03$ \\
\hline 120 & $10.12 \pm 0.55$ & $2.77 \pm 0.18$ & $0.86 \pm 0.07$ & $1.37 \pm 0.09$ \\
\hline
\end{tabular}

Values are Mean \pm S.E.M.; $\mathrm{n}=6$.

in neutrophil values, however, was obviously demonstrated at 600 and 1,200 mg/kg B.W. doses and was not found in any samples taken at 4 week- and 8 week-periods (data not shown). From such findings, it seems that long-term treatment (12 weeks) by fresh $S$. platensis would increase a neutrophil count, most likely in a dose-dependent manner. This effect of S. platensis has not been previously reported. In contrast to neutrophils, monocytes in each group were gradually increased from about $0 \%$ to 3-5\% in 12 weeks (Table 3). Their final numbers in both the control and treatment groups were quite similar, except for those of male rats treated with the low dose (300 mg/kg). Due to an unknown cause, five animals in this group showed such a remarkable increase in monocyte counts, ranging from 10 to $16 \%$, at the end of treatment (data not shown). The reason for neutrophil and monocyte stimulations by $S$. platensis treatment found in this study needs to be further elucidated. Both WBCs, however, are generally known as 'phagocytes'. They are responsible for innate immunity which provides the early lines of body defense. In general, neutrophils are promptly increased in response to an acute phase of inflammation, particularly as a result of bacterial infection, whereas chronic inflammation and necrosis can increase a monocyte count. As there was no appearance of infection or inflammation in our animals during the terminal period of study, a possibility that such elevation of neutrophils would be infection-and/or inflammation-associated effects, can be excluded. For the increase in monocytes, perhaps, it may be due to a body reaction to the continuing trauma caused by daily gavage and monthly blood sampling, since the monocyte levels in both control and treated animals were changed similarly. In addition, normal blood smears were observed in all of the animals. 
At autopsy, macroscopic observation of the organs did not show any abnormality in their gross appearances and weights (Table 4) due to the consumption of S. platensis. It should be also noted that there were no changes in morphology and no unusual lesions in the gastrointestinal tissues exposed directly to S. platensis. This provides the assurance that any possible accumulation of $S$. platensis resulting from prolonged exposure does not lead to toxicity. Similarly, there were no related effects on any of the gastrointestinal tract organs, the potential and direct target for toxic effects of foods. Since, pheophorbides, the breakdown products of chlorophyll by heat, are known to be toxic (Abrams, 1996), and sun-dried and cooked sun-dried diets of another blue-green algae, Scenedesmus acutus, have been previously reported to cause mild to moderate centrilobular fatty infiltration of the liver (Venkataraman et al., 1977) and a significant increase in liver weights (Pabst et al., 1978), we also tested S. platensis, dried under heat condition. In this study, no abnormalities, as evidenced by both blood chemistry values and the gross appearances of the internal organs, could be attributed to the feeding with oven-dried S. platensis.

We, then, have demonstrated that feeding S. platensis even at levels higher than any anticipated human consumption (300 mg/kg/day) (Becker, 1980) did not cause any undesirable effects to the experimental animals, and thus can be concluded to be not toxic. These results have provided additional data on the toxicology of spirulina and also offer more confidence that it can be safely used as a food and dietary supplement.

Acknowledgements This work was supported by the Royal Thai Government Budget (grant no. PHA 49127) and the Graduate School, Prince of Songkla University. Partial funding and graduate scholarship awarded to S. Satitit from the Academic Excellence Strengthening Program in Biochemistry of Prince of Songkla University, are also grateful. Authors wish to thank Assistant Professor Somsamorn Chittrakarn of Department of Pharmacology for her guidance of ocular bed puncture technique and Clinical Chemistry Unit at Songklanagarind Hospital for performing $\mathrm{Na}^{+}$and $\mathrm{K}^{+}$determinations. Deep appreciation also extends to Dr. Brian Hodgson for his suggestions and help in refining this manuscript.

\section{References}

Abrams, K.W. (1996). “Algae to Rescue!: Everything You Need to Know About Nutritional Blue-Green Algae.” Logan House, Studio City, USA.

Angkhasirisap, W., Inala, P., Sirimontaporn, A., Inpunkaew, R., Rungrojejinda, K., Kengkoom, K., Ratanasak, W. and Buripakdi Lawson, D. (2002). Blood chemistry profiles of outbred SpragueDawley rat in the Facility of National Laboratory Animal Care.
Presented at $28^{\text {th }}$ Congress on Science and Technology of Thailand, Bangkok, Thailand, October 26-30, p. 474.

Becker, E.W. (1980). Comparative toxicological studies with algae in India, Thailand, and Peru. In: “Algae Biomass Production and Use”, ed. by G. Shelef and C.J. Soeder. Elsevier/North-Holland, Amsterdam, Netherlands, pp. 565-574, 767-786.

Becker, E.W., Jakober, R., Luft, D. and Schmülling, R.M. (1986). Clinical and biochemical evaluation of the alga Spirulina with regard to its application in the treatment of obesity. A double-blind cross-over study. Nutr. Rep. Int., 33, 565-572.

Belay, A. (2002). The potential application of Spirulina (Arthrospi$\mathrm{ra}$ ) as a nutritional and therapeutic supplement in health management. J. Am. Nutraceut. Assoc., 5, 27-48.

Belay, A., Ota, Y., Miyakawa, K. and Shimamatsu, H. (1993). Current knowledge on potential health benefits of Spirulina. J. Appl. Phycol., 5, 235-241.

Bruce, R.D. (1985). An up- and down procedure for acute toxicity testing. Fundam. Appl. Toxicol., 5, 151-157.

Burtis, C.A., Ashwood, E.R. and Tietz, N.W. (1999). “Tietz Textbook of Clinical Chemistry.” W.B. Saunders, Philadelphia, USA.

Casey, J.D. and King, D.J. (1980). Clinical chemical values for some common laboratory animals. Clin. Chem., 26, 1877-1879.

Chamorro, G., Salazar, M., Izquierdo, E., Salazar, S. and Ulloa, V. (1985). Multi-generation study on reproduction and lactation in rats fed Spirulina. Archiv. für Hydrobiologie, 20, 165-171.

Chamorro, G., Salazar, M. and Pages, N. (1996). Dominant lethal study of Spirulina maxima in male and female rats after shortterm feeding. Phytother. Res., 10, 28-32.

Chamorro. G., Salazar, S., Favila-Castillo, L., Steele, C. and Salazar, M. (1997). Reproduction and peri- and postnatal evaluation of Spirulina maxima in mice. J. Appl. Phycol., 9, 107-112.

Chamorro, G.A., Herrera, G., Salazar, M., Salazar, S. and Ulloa, V. (1988). Short-term toxicity study of Spirulina in F3b generation rats. J. Toxicol. Clin. Exp., 8, 163-167.

Charmorro, G., Salazar, M., Favila, L. and Bourges, H. (1996) Pharmacology and toxicology of Spirulina alga. Rev. Invest. Clin., 48, 389-399.

de Mule, M.C., de Caire, G. and de Cano, M. (1996). Bioactive substances from Spirulina platensis (Cyanobacteria). Int. J. Experiment. Bot., 58, 93-96.

Fox, R.D. (1996). “Spirulina: Production and Potential.” Edisud, Aix-en-Provence, France.

Inala, P., Sirimontaporn, A., Inpunkaew, R., Rungrojejinda, K., Kengkoom, K., Ratanasak, W. and Buripakdi Lawson, D. (2002). Hematological analysis of outbred Sprague-Dawley rat in the Facility of National Laboratory Animal Centre. Presented at $28^{\text {th }}$ Congress on Science and Technology of Thailand, Bangkok, Thailand, October 26-30, p. 475.

Iwata, K., Inayama, T. and Kato, T. (1990). Effects of Spirulina platensis on plasma lipoprotein lipase activity in fructose-induced 
hyperlipidemia in rats. J. Nutr. Sci. Vitaminol., 36, 165-171.

Jassby, A. (1988). Spirulina: a model for microalgae as human food. In: “Algae and Human Affairs", ed. by C. Lembi and J.R. Waaland. Cambridge University Press, Cambridge, UK, pp. 149-179.

Khan, Z., Bhadouria, P. and Bisen, P.S. (2005). Nutritional and therapeutic potential of Spirulina. Curr. Pharm. Biotechnol., 6, 373-379.

Lacaz-Ruiz, R. and Mos, E.N. (1990). Produçao de biomassa de Spirulina maxima para alimentaçao humana e animal. Revista de Microbiologia, 21, 85-97 (in Spanish).

Lightner, D.V. (1987). Possible toxic effects of the marine bluegreen alga, Spirulina subsala, on the blue shrimp, Penaeus styrostris. J. Invertebr. Pathol., 32, 139-150.

Pabst, W., Payer, H.D., Rolle, I. and Soeder, C.J. (1978). Multigeneration feeding studies in mice for safety evaluation of the microalga, Scenedesmus acutus. I. Biological and haematological data. Food Cosmet. Toxicol., 16, 249-254.

Parada, J.L., de Caire, G., de Mule, M.C. and de Cano, M.M. (1998). Lactic acid bacteria growth promoters from Spirulina platensis.
Int. J. Food Microbiol., 45, 225-228.

Raza, J.A., Babb, J.D. and Movahed, A. (2004). Optimal management of hyperlipidemia in primary prevention of cardiovascular disease. Int. J. Cardiol., 97, 355-366.

Salazar, M., Chamorro, G.A., Salazar, S. and Steele, C.E. (1996). Effect of Spirulina maxima consumption on reproduction and peri- and postnatal development in rats. Food Chem. Toxicol., 34, 353-359.

Salazar, M., Martinez, E., Madrigal, L., Ruiz, L.E. and Chamorro, G.A. (1998). Subchronic toxicity study in mice fed Spirulina maxima. J. Ethnopharmacol., 62, 235-241.

Tsuchihashi, N., Watanabe, T. and Takai, Y. (1987). Effect of Spirulina platensis on caecum content in rats. Bull. Chiba Hygiene College, 5, 27-30.

Venkataraman, I.V., Becker, W.E., Khanum, P.M. and Mathew, K.R. (1977). Short-term feeding of alga Scenedesmus acutus processed by different method: growth pattern and histological studies. Nutr. Rep. Int., 16, 231-240. 